\title{
Educação em saúde: a orientação alimentar através de atividades de grupo
}

\author{
Health education: dietary guidance through group activities \\ Educación en salud: la orientación alimentar a través \\ de actividades de grupo
}

Rosana Maffacciolli', Marta Julia Marques Lopes²

\begin{abstract}
RESUMO
Trata-se de um projeto assistencial que visou à implantação de um grupo com enfoque na re-educação alimentar e na qualidade de vida no âmbito de um Programa de Saúde da Família de Porto Alegre. O objetivo dessa proposta foi a introdução de práticas de grupo orientadas para a educação-prevenção de danos à saúde, centradas no componente alimentar, através de dinâmicas de grupo. As atividades de grupo foram descritas em diário de campo o que possibilitou contemplar não somente as especificidades objetivas dessas ações, mas também o caráter reflexivo das dinâmicas e dos depoimentos colhidos. Portanto, pôde-se visualizar a assistência sob o foco educativo, apoiando-se em elementos como a promoção das relações interpessoais que convergem para o estabelecimento de confiança e autodeterminação. Dessa forma, investiu-se na criação de um ambiente favorável para a revisão de comportamentos e condicionamentos decorrentes dos mesmos.
\end{abstract}

Descritores: Prática de grupos; Serviços básicos de saúde; Educação em saúde

\begin{abstract}
This is an assistance project for the implementation of a group focusing dietary reeducation and life quality within 'Programa de Saúde da Familia de Porto Alegre', a family health program. The objective of this proposal was the introduction of group practices to both educate on and prevent health disorders associated with feeding through group dynamics. Group activities have been described in a field diary. This enabled the research team to contemplate not only the particular specifics of actions but also the reflective character triggered by the dynamics and reports collected. Therefore, assistance could be viewed under an educational focus, relying on elements such as the promotion of interpersonal relationships which converge to the establishment of trust and self-determination. In this way, there was an investment in the development of a favorable environment for the revision of behaviors and conditionings.
\end{abstract}

Keywords: Group practice; Basic health services; Health education

\section{RESUMEN}

Se trata de un proyecto asistencial que visó a la implantación de un grupo con enfoque en la reeducación alimentar y en la cualidad de vida en el ámbito de un Programa de Salud da Familia de Porto Alegre. El objetivo de esa propuesta fue la introducción de prácticas de grupo orientadas para la educación-prevención de daños a la salud, centradas en el componente alimentar, a través de dinámicas de grupo. Las actividades de grupo fueron descritas en diario de campo lo que posibilitó contemplar no solamente las especificidades objetivas de esas acciones, sino el carácter reflexivo de las dinámicas y testigos recogidos. Por lo tanto, se puede visualizar la asistencia bajo el foco educativo, apoyándose en elementos como la promoción de las relaciones interpersonales que convergen para el establecimiento de confianza y autodeterminación. De esa forma, se invistió en la creación de un ambiente favorable para la revisión de comportamientos y condicionamientos decurrentes de los mismos.

Descriptores: Practica de grupo; Servicios básicos de la salud; Educación en salud

\footnotetext{
1 Mestranda em Enfermagem da Universidade Federal do Rio Grande do Sul - UFRGS - Rio Grande do Sul(RS),Brasil.

2 Professora Titular do Departamento de Assistência e Orientação Profissional da Universidade Federal do Rio Grande do Sul UFRGS - Rio Grande do Sul (RS),Brasil.
} 


\section{INTRODUÇÃO}

O desenvolvimento desse artigo partiu da experiência e da redação prévia de trabalho de conclusão de curso de graduação em enfermagem da Escola de Enfermagem da Universidade Federal do Rio Grande do Sul em 2003. O referido trabalho versou sobre uma experiência de implementação de um grupo de re-educação alimentar e qualidade de vida em uma unidade do Programa de Saúde da Família (PSF) Pitoresca, situado no bairro Jardim Bento Gonçalves do município de Porto Alegre - RS.

A proposta, caracterizada como projeto assistencial, dirigiu-se a pacientes adultos e idosos acompanhados em nível ambulatorial. Essa população estava inserida previamente numa proposta de atendimento ambulatorial, abrangendo, principalmente, Consulta de Enfermagem, intitulada Ambulatório de Promoção da Qualidade de Vida -APQV. Tal proposta consiste em desenvolver estratégias assistenciais e educativas, visando influenciar hábitos de vida para a promoção da saúde e da qualidade de vida. Originou-se a partir de uma modalidade de pesquisa-desenvolvimento intitulada "As doenças crônicodegenerativas e a promoção da qualidade de vida" que compõe a Rede de Metodologias de Educação e Assistência na Promoção da Qualidade de Vida / Plano Sul de Pesquisa e Pós-Graduação, financiado pelo CNPq e coordenado pela professora Marta Júlia Marques Lopes da Escola de Enfermagem - UFRGS.

O foco desta abordagem está centrado na cultura alimentar e na reeducação alimentar, particularmente. Os usuários cadastrados no APQV são sistematicamente acompanhados através de Consulta de Enfermagem com agendamento regular e eletivo. Dentre alguns pressupostos que embasam a Consulta de Enfermagem, na perspectiva do APQV, podem-se destacar o estabelecimento de empatia, a escuta ativa, a confiança, a aceitação e ausência de julgamento como pressupostos relacionais para otimizar a adesão às terapias e maior envolvimento entre enfermeira e paciente ${ }^{(1)}$.

A proposta de implementação de grupos, buscando a complementaridade terapêutica, surgiu a partir da necessidade do APQV e do PSF de oferecer uma modalidade terapêutica mais abrangente, no sentido de promover educação em saúde aos usuários com conseqüente reflexo na diminuição de demanda ambulatorial cotidiana, especialmente de pacientes portadores de danos de longa duração decorrentes da hipertensão, diabetes, obesidade. Nesse contexto, a temática "hábitos alimentares", muitas vezes, configura-se na razão que leva esses usuários ao atendimento individual.

Pensa-se que, agregados em grupo e estabelecidos os objetivos que os une, os indivíduos se posicionam em função da coletividade, reformulam-se regras a despeito de compreensões singularmente incorporadas. A experiência do grupo atua sobre nós de tal modo que chega a modificar nossos hábitos, situações de trabalho e, ainda, nossos objetivos de vida.

Nessa linha de raciocínio, propôs-se essa modalidade de assistência, consciente de que, além de desenvolver a integralidade assistencial, valorizou essencialmente a atuação da enfermeira enquanto educadora na área da saúde.

\section{AASSISTÊNCIA EM GRUPOS COMO FERRAMENTA TERAPÊUTICA}

Divulga-se amplamente na mídia, e o conhecimento científico estabelece nexos complexos entre hábitos de vida e o desenvolvimento de fatores de risco, que condicionam agravos à saúde. Entre esses fatores, sobressaem a alimentação inadequada (causando, principalmente a obesidade), o sedentarismo, as situações de estresse emocional, entre outros. O reflexo dessas condições resulta, em termos epidemiológicos, no desenvolvimento das principais patologias associadas às situações de risco citadas acima. As doenças cardiovasculares, por exemplo, constituíram-se na primeira causa de morte no Brasil em 1998 (27,4\% dos óbitos) e, no ano de 2000, constatou-se cinco milhões de pessoas com diagnóstico de diabetes mellitus ${ }^{(2)}$.

As características desses agravos à saúde requerem um acompanhamento sistemático e contínuo, já que não há resolução do problema, mas a possibilidade de se viver melhor e com qualidade ${ }^{(3)}$. Portanto, torna-se necessário investir na reeducação alimentar, na prática de atividade física, na interação em atividades de grupo e lazer, entre outros, para intervir positivamente nesses processos patológicos.

Nesse sentido, reconhecendo as implicações dos hábitos prejudiciais à saúde, faz-se necessário o estabelecimento de estratégias que influenciem mudanças comportamentais e, por conseguinte, o controle e prevenção de doenças ligadas ao estilo de vida. Dentre essas estratégias, pode-se destacar as dinâmicas de grupo. Esse tema é evidenciado ${ }^{(4)}$ como possibilidade de multiplicação dos recursos assistenciais e construção de novos e eficazes modelos que possam ser mais abrangentes, multidimensionados, de maior alcance e de mais fácil acessibilidade.

A deficiência da adesão dos pacientes às orientações que precisam ser seguidas para controlar sinais e sintomas das doenças, pode ser relacionada ao fato de que as interações entre usuários e prestadores de serviços em saúde se configuram, atualmente, de forma superficial, em termos comunicacionais. $O$ fato é que não existe comunicação totalmente objetiva. Esta se faz entre pessoas e cada pessoa é um mundo à parte, com seu subjetivismo, suas experiências, sua cultura, seus valo- 
res, seus interesses e suas expectativas. A percepção pessoal funciona como uma espécie de filtragem que condiciona a mensagem segundo a própria lente. Ouvimos e vemos conforme a nossa percepção ${ }^{(5)}$.

Portanto, acredita-se que, considerar os sujeitos a partir de suas condições objetivas, valores e experiências socioculturais, além de reforçar a confiabilidade mútua, implica uma conveniente compreensão das informações prestadas; deve-se analisá-los como a medida e a referência para as ações e estratégias preventivas que lhes são dirigidas ${ }^{(6)}$.

Nesse sentido, a comunicação intermediada através das atividades de educação em saúde pode ser vista como um mecanismo de intercâmbio entre o saber científico e o popular, proporcionando a socialização do saber científico e o reconhecimento do saber popular. Constitui-se em meta de atuação que possibilita, a médio e a longo prazos, que o indivíduo exerça maior prevenção e controle de doenças as quais possam vir a instalar-se e que a coletividade seja um agente organizado de intervenção sobre os determinantes imediatos e estruturais do processo saúde-doença ${ }^{(7)}$.

Somado a isso, o formato de grupo possibilita o senso de inclusão, valorização e identificação entre os participantes, sendo que muitos buscam amparo diante de seus problemas de saúde. Nesse aspecto, pode-se estabelecer uma relação metafórica ao comparar a constituição de um grupo com uma galeria de espelhos, onde cada participante pode refletir e ser refletido nos e pelos outros, oportunizando a discriminação, afirmação e consolidação de suas próprias identidades ${ }^{(4)}$. Desenvolve-se uma relação onde cada indivíduo vê-se acolhido: a situação do outro reflete a sua situação pessoal. A partir disso, constitui-se uma base de sustentação e fortalecimento de relações, em que as vivências individuais transformam-se em aprendizado.

O trabalho com grupos proporciona o aprofundamento de discussões pelas quais consegue-se ampliar conhecimentos e melhor conduzir o processo de educação em saúde, de modo que as pessoas possam superar suas dificuldades, obtendo maior autonomia e podendo viver mais harmonicamente com sua condição de saúde ${ }^{(8)}$.

É consenso que somente a mudança no estilo de vida provoca reflexos, tanto preventivos quanto curativos a portadores de agravos à saúde de longa duração como é o caso do sobrepeso/obesidade e as comorbidades relacionadas. Torna-se viável, portanto, a adoção de recursos adjuvantes no cotidiano assistencial dos serviços de saúde, especialmente nessas condições em que se observa que a influência ambiental em muito sobrepuja a intencionalidade de autocuidado entre as pessoas.

\section{A ESTRATÉGIA: AS ATIVIDADES COM O GRUPO}

As atividades desenvolvidas na proposta descrita foram mencionadas em conformidade com um modelo de diário de campo adotado no decorrer das análises o qual serviu como elemento direcionador durante as observações. Dessa maneira, as atividades e observações foram descritas de forma a contemplar não somente as especificidades objetivas dessas ações, mas também o caráter reflexivo das análises colhidas.

\section{Espaço e Tempo das Atividades}

O tempo e as datas estipuladas para a realização dos grupos, inicialmente sofreram alterações, pois durante os dois primeiros encontros, o grupo permaneceu subdivido em atividades que aconteciam nas segundas e terças-feiras. O objetivo consistia em contemplar todos os participantes que haviam sido convidados. Contudo, percebeu-se que, em média, seis pessoas compareciam por reunião, e decidiu-se formar somente um grupo o qual prevaleceu nas reuniões seguintes.

O local das reuniões foi um dos consultórios do PSF Pitoresca. Nesse consultório, são realizadas as Consultas de Enfermagem do APQV, o que representou uma certa familiaridade para os participantes. Contudo, objetivando acomodar a todos, era realizada, antes do início dos encontros, uma conformação mais acolhedora onde cadeiras se distribuíam centralmente; uma maca que ali se localizava era removida para outro setor do posto; uma mesa era disposta em uma das laterais da sala e preparavam-se chás e biscoitos à disposição das pessoas.

\section{O grupo/componentes e motivações}

O grupo formou-se a partir da demanda ambulatorial da Consulta de Enfermagem para re-educação alimentar preconizada pelo APQV. Todos os aspectos tratados na consulta individual passaram, nesse momento, a ser retomados coletivamente. Evidencia-se na literatura ${ }^{(4)}$ a pertinência desse tipo de abordagem para o tratamento da obesidade/sobrepeso, a qual pode ser caracterizada de grupos terapêuticos. Nesse contexto, existe um objetivo de mudança comportamental que culmina em mudanças pessoais significativas, incluindo, ainda, procedimentos de auto-ajuda e educacionais, os quais constituem tendências muito utilizadas para o tratamento de transtornos alimentares.

Quanto aos participantes, alguns apresentavam obesidade e outros já haviam atingido a meta estabelecida em consultório para redução de peso, mas optaram por continuar participando das orientações. 
Nos encontros iniciais, questionou-se junto aos participantes sobre as motivações que os traziam até o grupo. A maioria das pessoas relatou sobre suas condições de obesidade e/ou sobrepeso e quanto à intenção em buscar uma resolução para esses problemas, já que, essas situações os prejudicavam no desenvolvimento das tarefas rotineiras, profissionais e, até mesmo em relação ao convívio familiar. Evidenciou-se, por vezes, em algumas falas, condutas passivas em relação aos próprios problemas, onde as pessoas descreviam sua condição de saúde e o quanto essa se encontrava prejudicada. Um depoimento ressaltou essa idéia quando um dos participantes declarou não visualizar sua forma física de modo negativo, somente a condição "deletéria" à saúde em consequiência da obesidade o incomodava. Para outras pessoas, no entanto, sobressaíam os aspectos da socialização, da aquisição de aprendizado e a oportunidade de se envolverem em diálogos acerca dos assuntos em pauta.

Ocorre que essas concepções não são incomuns entre as pessoas que participam de atividades de grupo. Em um estudo ${ }^{(9)}$ sobre a articulação entre educação e saúde no âmbito de um programa de saúde escolar, pelo qual, entre outras práticas assistenciais se efetivavam grupos, as famílias destacavam a relevância dessas atividades, tomando-as como momentos oportunos para se discutir diversos assuntos pertinentes à criança e ao comportamento infantil.

Pôde-se vislumbrar, dessa forma, que as diferentes motivações levantadas encontraram novamente sustentação na literatura ${ }^{(4)}$ sobre os objetivos e métodos que podem ser desenvolvidos nesses grupos. As informações são importantes para o compartilhamento de noções básicas sobre a doença, seu processo terapêutico e, amplamente, quanto a noções sobre o próprio funcionamento do grupo. Contudo, deve-se atentar para que essas informações se processem de forma clara e participativa, pois a incerteza sustentada é um fator ansiogênico que poderá ser perturbadora para os ouvintes.

\section{As Atividades e Atitudes no Grupo}

As técnicas de animação propiciaram momentos de descontração e interação entre os participantes, fornecendo subsídios para a adesão e participação ativa dos integrantes nas temáticas desenvolvidas. As oficinas propiciaram um método para viabilizar o labor da investigação e incorporar o significado dos atos e as relações com as estruturas sociais ${ }^{(10)}$. Ainda, tiveram o objetivo de promover a construção do conhecimento de forma contextualizada e autogestionada.

As atividades realizadas nos grupos incluíram debates sobre temas estabelecidos entre os participantes e a equipe. Apresentavam-se as temáticas alternativamente e decidia-se sobre a pauta para as reuniões subseqüen- tes. Os encontros constituíam-se por meio de divulgação de informações prestadas pela equipe; os participantes opinavam de acordo com suas compreensões acerca do assunto e, nesses momentos, propunham-se técnicas para dinamizar o envolvimento interpessoal. Em relação a essa forma de direcionar a atividade grupal, é recomendável $^{(7)}$ que se abra mão de dinamismos formais, sendo mais adequada a utilização de abordagens que envolvam movimento, como teatro, painéis de colagem, desenhos, jogos, audiovisuais, entre outros.

Sendo assim, nos primeiros encontros, com a pretensão de iniciar a apresentação dos participantes de forma lúdica, utilizaram-se algumas técnicas que levaram em consideração esse intento. No transcorrer das atividades seguintes, foram sendo incluídos os temas definidos conforme o contexto grupal e realizadas as dinâmicas as quais eram planejadas para abrangê-los, como seguem:

Dinâmica 1 - os participantes acomodavam-se em suas cadeiras que eram dispostas em círculo; uma das pessoas iniciava a atividade identificando-se e atribuindo-se uma qualidade, contendo a mesma letra pela qual inicia seu primeiro nome. Todos permaneciam revelando seus nomes e as qualidades eleitas, considerando que a pessoa que se apresentasse posteriormente, deveria repetir a fala anterior, até a manifestação completa dos participantes. Reconheceu-se, em algumas pessoas, comportamentos inibidos que dificultaram a tarefa de atribuírem-se qualidades, sendo que o restante do grupo, diante disso, apontava alternativas para auxiliá-los. Outros, entretanto, mais extrovertidos, referiam qualidades que denotavam aspectos descontraídos e, com isso, provocavam risos nos demais participantes. Essa dinâmica permitiu subsidiar a memorização dos nomes das pessoas, pois foi facilitada através da inclusão das qualidades atribuídas, sendo essas lembradas pelos integrantes em outros momentos grupais.

Dinâmica 2 - ao se tratar sobre obesidade e alimentação, os participantes teriam de expressar duas dúvidas e duas certezas a respeito do assunto. Quatro pedaços de papel e uma caneta foram fornecidos para que, individualmente, pudessem escrever o que fora solicitado. Após, os papéis foram dobrados e depositados em uma urna, a qual, ao som de uma música, percorria por todos. Ao cessar a música, a pessoa que ficasse com a urna deveria retirar um dos bilhetes depositados e ler seu conteúdo. Em sendo uma dúvida, dever-se-ia responder e se fosse uma certeza, dever-se-ia opinar.

Essa atividade propiciou uma discussão bastante relevante, pois tornou possível, a partir de avaliações emitidas pelos próprios componentes do grupo, elucidar algumas crenças sobre julgamentos erroneamente incorporados e elaborar adaptações que levassem em conta comportamentos mais adequados em detrimento das condições nocivas à saúde. 
Dinâmica 3 - propôs-se trabalhar com as recomendações abordadas em nível ambulatorial. Foram apresentados uma série de desenhos os quais retratavam situações relacionadas ao tema: figuras de alimentos, pessoa verificando seu peso, equivalência entre as refeições, alimentos hipercalóricos, realização de atividade física, entre outros. Essas ilustrações eram distribuídas aos participantes que deveriam expor seus entendimentos diante do que lhes era exibido. Como na proposta anterior, foram exploradas as noções relativas ao componente comportamental. Paralelamente, em muitos momentos, o desenvolvimento dessa atividade conduziu-se por identificações situacionais, onde os participantes se espelhavam em determinadas imagens que lhes remetiam ao enfrentamento dos seus problemas.

Abordou-se, em outro momento, a importância dos exercícios físicos para a saúde. Inicialmente, decidiu-se argumentar a partir das implicações que o estabelecimento desse hábito representa como fator imprescindível de uma vida mais saudável. Antes, porém, para que voltassem suas atenções para o assunto, os participantes foram indagados sobre o significado da palavra sedentarismo. Uma pessoa, então, referiu que sedentarismo exprime: "não fazer nada e só ficar em casa". Explicou-se que o sentido desse termo era o oposto de praticar atividade física e que essa prática, por sua vez, somente é considerada como tal se realizada três vezes por semana ou todos os dias pelo tempo de, no mínimo, 30 minutos. Tarefas diárias como os trabalhos domésticos e visitas aos supermercados não poderiam ser classificados como atividades físicas regulares.

Os benefícios, somados ao efeito na redução de peso, foram citados: diminuição dos níveis de pressão arterial e riscos cardiovasculares; redução das doses, ou mesmo a descontinuidade no uso de medicações antihipertensivas e hipoglicemiantes; menor probabilidade no desenvolvimento de diabetes mellitus; adequação dos níveis de colesterol e triglicerídeos; melhora das condições psicológicas, amenizando episódios depressivos e de ansiedade (explicou-se sobre o mecanismo das endorfinas definindo-as como substâncias produzidas no organismo em resposta a experiências prazerosas). Após essas explanações, reproduziu-se um filme educativo desenvolvido pelo Ministério da Saúde, intitulado "Atividade física e saúde" no qual aparecem pessoas envolvidas em atividades físicas e prestando depoimentos que corroboram com as informações relacionadas ao tema. Esses comentários propiciaram aos participantes cogitarem a articulação de agrupamentos de pessoas com a intenção de promoverem caminhadas em áreas disponíveis no bairro. Ao mesmo tempo, refletiu-se no encorajamento de um dos participantes em iniciar, de acordo com o seu relato, atividades físicas e promovê-las junto a sua família.
No decorrer dos encontros, os aspectos emocionais foram retratados através dos diálogos. Ao se tratar sobre hábitos alimentares, principalmente, muitas declarações refletiam atitudes impotentes frente à compulsão de ingerir alimentos, geralmente hipercalóricos. A ocorrência dessas considerações proporcionou um dia de reunião do grupo para abordagem do assunto "ansiedade e depressão". Em princípio, perguntou-se para os presentes se algum deles já teria vivenciado situações de ansiedade e todos responderam positivamente. $\mathrm{O}$ médico que desenvolvia o assunto afirmou então, que $100 \%$ das pessoas experimentam essa sensação e que ela está relacionada à idéia de reação-fuga. A depressão, por outro lado, correlaciona-se a reações não responsivas diante de determinadas experiências.

$\mathrm{Na}$ seqüência, alguns sintomas foram apresentados como característicos dessas condições psicológicas. A ansiedade provoca aceleração dos batimentos cardíacos e dificuldade em iniciar o sono; na depressão existe um desconforto torácico que não está relacionado aos batimentos cardíacos, mas sim a uma sensação de "vazio". Também, aqui, o padrão de sono é prejudicado, pois existe dificuldade da permanência nesse estado, ocorrendo despertar precoce durante a madrugada.

Por fim, foram convencionados três pensamentos básicos aos quais deve-se recorrer diante dessas situações. O primeiro deles deve partir da procura de elementos para o autoconhecimento; estar consciente de que nem tudo e nem todos dispõem de perfeição e, acima de tudo, estimar a si próprio.

\section{Os Conflitos no Convívio Grupal}

Mesmo implícitas, as situações de conflito, em determinados momentos, permearam o convívio grupal. As questões sobre as quais esses conflitos estiveram relacionados assentaram-se sobre os objetivos do grupo, papéis desempenhados pelos participantes e sobre a coesão grupal.

Quanto aos objetivos, evidenciou-se, na forma de participação de um dos componentes do grupo, resistência em aceitar as propostas de mudança comportamental ou de atitude. As alternativas para a busca de um estilo de vida pautado em atitudes menos passivas frente às adversidades, não se faziam úteis, ao contrário, no seu entendimento, eram consideradas enfadonhas e sem credibilidade. Notou-se que esse comportamento influenciou o andamento dos assuntos que estiveram em discussão e poderia induzir negativamente a compreensão pretendida através das dinâmicas.

Nesse contexto, a resistência a mudanças pode apresentar-se como um processo naturalmente presente nas relações humanas. O investimento e esforço para o abandono de hábitos arraigados e atitudes presentes na for- 
ma de enfrentamento com o cotidiano podem despertar sentimentos de ambivalência que serão tão mais intensos quanto maior for a dificuldade em vulnerabilizar-se para a aventura do novo e da mudança.

Houve momentos de frustração da equipe em relação a determinadas condutas. Muitas vezes, o assunto que estava sendo tratado era desviado para conversas colaterais que destoavam das considerações em andamento. Isso acarretava sentimento de decepção no coordenador à medida que eram entendidos como desvalorização do trabalho e estagnação no processo produtivo do grupo.

Em relação a esse ponto de conflito, faz-se necessário remeter-se aos atributos desejáveis para um coordenador de grupo ${ }^{(4)}$. Nesse caso, destaca-se a capacidade negativa. Essa função consiste na condição de um coordenador de grupo de conter as suas próprias angústias de forma que não invadam todo o espaço de sua mente. Caso contrário, terá de trabalhar sob um considerável desgaste.

A avaliação da coesão grupal mostrou que, não houve total entrelaçamento do grupo. Essa constatação pode estar ligada ao fato de que o grupo permaneceu subdividido em duas frentes nas datas iniciais de realização das atividades. Contudo, acreditou-se que essa circunstância poderia desintegrar-se no decorrer de futuros encontros, já que se verificou, a partir de apreciações positivas, a mobilização dos participantes para a preservação do grupo. É iminente o estabelecimento da coesão grupal, na direção do estabelecimento de um certo grau de proximidade e honestidade entre as pessoas ${ }^{(11)}$. Essas premissas permeiam e consolidam o grupo como um todo permitindo aos integrantes sentirem-se seguros para investirem verdadeiramente na proposta grupal.

\section{Atividades de Avaliação e Fechamento}

Com intuito de resgatar as proposições trabalhadas, na fase final das reuniões, foram desenvolvidas algumas atividades relacionadas aos temas que foram trabalhados em cada momento.

Em um dos primeiros dias de encontro, no qual trabalhou-se com a apresentação dos participantes e quanto as suas motivações em ingressar no grupo, a atividade de encerramento consistiu na leitura de uma mensagem intitulada "Chapéu roxo!". Descrevia o ciclo de vida de uma pessoa que ao observar-se em um espelho, atribuía-se muitas imperfeições físicas. Ao passo que envelhecia, entretanto, foi abandonando essa atitude e permitiu-se observar mais atentamente a vida que a envolvia. Quando, aos 80 anos, deparou-se com a sua imagem em frente ao espelho, decide vestir um chapéu roxo e sair para se divertir com o mundo. A mensagem é finalizada com a seguinte frase: "talvez todos nós devamos pegar nosso chapéu roxo um pouquinho mais cedo... e aproveitar mais cada fase de nossas vidas". Consentiu-se, com essa leitura, a reflexão sobre o quanto as pessoas se envolvem com as ocorrências supérfluas do cotidiano e quanto à brevidade que, com esse comportamento, se institui à vida.

$\mathrm{Na}$ ocasião em que se tratava sobre atividades físicas, definiu-se que seria vantajoso ensinar aos participantes exercícios de alongamento. Anteriormente, falouse sobre a importância dessa prática explicando que ela deveria anteceder e suceder à realização do exercício físico. Após, ao som de uma música, os participantes posicionavam-se em pé e, seguindo as orientações da equipe, alongaram os músculos dos braços, pernas e dorso. Mesmo com certas dificuldades, constatou-se a participação de todos e o interesse em que essa abordagem fosse repetida em outras ocasiões.

Em relação ao encontro que tratou da questão da ansiedade e depressão, trabalhou-se com uma técnica de relaxamento. Primeiramente, argumentou-se sobre a adoção dessa estratégia nos momentos críticos como uma alternativa acessível de instituir o autocontrole. Essa técnica abrangeu exercícios de respiração nos quais as pessoas, dispostas confortavelmente nas cadeiras, deveriam posicionar suas mãos sobre o abdome. Na inspiração, haveria expansão torácica e na expiração, a expansão abdominal, prolongando o máximo possível essa fase.

Durante estes exercícios respiratórios, para atingir eficientemente o relaxamento, utilizou-se uma música proveniente da região da Cordilheira dos Andes. Propositalmente, esse lugar fora descrito especificando-se as civilizações antigas que lá habitavam e sobre a magnitude das ruínas em meio à natureza exuberante. Os participantes, de olhos fechados, foram convidados a imaginarem a região remetendo-se a ela. Teriam uma visão de cima das montanhas e observariam a imensidão do céu em meio ao misticismo que compreende a região. Após 10 sequiências de respiração, finalizou-se a dinâmica. Sempre que se deparassem com situações de estresse emocional e se fosse possível, poder-se-ia recorrer a essa tática, não desconsiderando a imposição de pensamentos agradáveis. Essa condição é significativa na prática de atividades de relaxamento, pois o pensamento arrolado a situações de satisfação ou a visualização de cenários que causem sensação de tranqüilidade, estimulam mecanismos neurológicos de indução na produção de substâncias relacionadas ao prazer ${ }^{(12)}$.

A avaliação do grupo e o estabelecimento das temáticas para os encontros seguintes também constituíram a prática de finalização das reuniões. Nessas ocasiões foi possível ponderar sobre a validação das ações grupais a partir da participação e aprovação dos integrantes nas atividades sugeridas. 


\section{CONSIDERAÇÕES FINAIS}

Ao final desse trabalho, constatou-se que, no meio grupal, é possível reconhecer como aspecto imprescindível um espaço de socialização que promove suporte emocional. Além disso, implicitamente, constatou-se o potencial efeito de estimular atitudes criativas em meio às dinâmicas. Muitas vezes, os próprios participantes, conforme suas falas, surpreendiam-se diante de suas aptidões nos momentos de interação em grupo.

Por outro lado, é conveniente salientar que essa proposta configurou uma ação de implantação e como tal, vários aspectos podem ser retomados em outras oportunidades no esforço de aperfeiçoar esse tipo de assistência.

Esse recurso também favoreceu a introdução dos elementos da equipe institucional nas especificidades do cotidiano vivencial dos pacientes. Em se tratando de um ambiente promotor de expressões individuais, torna-se um campo promissor para o desenvolvimento da reciprocidade entre a assistência e os atores a que se dirige. $\mathrm{O}$ que se configura, então, a partir de propostas como esta, é a possibilidade de se recriar posicionamentos ambivalentes no sentido da aproximação relacional entre terapeutas e pacientes e resgatar a empatia no sentido de reestruturar as ações de prevenção em saúde.

\section{REFERÊNCIAS}

1. Lopes MJM, Silveira DT, Ferreira SRS. Educação em saúde nas doenças crônico-degenerativas e a promoção da qualidade de vida. Estudos Interdisciplinares sobre o Envelhecimento.1999; 2:121-30.

2. Brasil. Ministério da Saúde. Secretaria de Políticas de Saúde. Plano de reorganização da atenção à hipertensão arterial e diabetes mellitus. Brasília(DF): Ministério da Saúde; 2002.

3. Porto GB. Palavras desenhadas - Um protocolo de orientação alimentar através de desenhos na consulta de enfermagem [Trabalho de Conclusão de Curso]. Porto Alegre (RS): Universidade Federal do Rio Grande do Sul: UFRGS; 2002.

4. Zimerman DE, Osório LC, organizadores. Como trabalhamos com grupos. Porto Alegre: Artes Médicas; 1997.

5. Silva MJP. Comunicação tem remédio: a comunicação nas relações interpessoais em saúde. São Paulo: Gente; 1996.

6. Brasil. Ministério da Saúde. Secretaria de Políticas de Saúde. Manual de redução de danos. Brasília(DF): Ministério da Saúde; 2001.

7. Duncan BB, Shimidt MI, Giugliani ERJ. Medicina ambulatorial - condutas clínicas em atenção primária. Porto Alegre: Artes Médicas; 1996.
8. Silva DGV, Francioni FF, Natividade MSL, Azevedo M, Sandoval RCB, Lourenzo VM. Grupos como possibilidade para desenvolver educação em saúde. Texto \& Contexto Enferm. 2003; 12(1): 97-103.

9. Ferriani MGC, Ubeda EML. Articulação: educação e saúde. A percepção dos atores sociais que utilizam o Programa de Assistência Primária de Saúde Escolar (PROASE) no município de Ribeirão Preto. Acta Paul Enferm. 1998; 11(1): 46-55.

10. Araújo MFM, et al. Port Folio : como trabalhamos com o método de oficinas. Fortaleza: Universidade Federal do Ceará; 1998.

11. Munari DB. Enfermagem e grupos. 2a ed. Goiânia: AB; 2003.

12. Pastore K. A mais comum das dores. Revista Veja. 2003; 36 (27): 65-78. 